M. Alama-Bućko, A. V. Nagaev and A. Zaigraev (Toruń)

\title{
ASYMPTOTIC ANALYSIS OF MINIMUM VOLUME CONFIDENCE REGIONS FOR LOCATION-SCALE FAMILIES
}

Abstract. An asymptotic analysis, when the sample size $n$ tends to infinity, of the optimal confidence region established in Czarnowska and Nagaev (2001) is considered. As a result, two confidence regions, both close to the optimal one when $n$ is sufficiently large, are suggested with a mild assumption on the distribution of a location-scale family.

1. Introduction. Let $x_{1}, \ldots, x_{n}$ be independent copies of a random variable $\xi$. Suppose that the distribution function of $\xi$ belongs to a parametric family $\mathcal{F}=\left\{F: F=F_{\theta}, \theta \in \Theta \subset \mathbb{R}^{d}\right\}, d \geq 2$. The unknown parameter $\theta$ is to be estimated on the basis of the sample $x=\left(x_{1}, \ldots, x_{n}\right)$.

Let $\left(\mathbb{R}^{n}, \mathcal{B}^{n}, \mathrm{P}_{\theta}\right)$ be the sample space induced by $x=\left(x_{1}, \ldots, x_{n}\right)$ and $F_{\theta} \in \mathcal{F}$ where $\mathcal{B}^{n}$ is the $\sigma$-algebra of Borel subsets of $\mathbb{R}^{n}$.

Two approaches to parameter estimation are known: the point estimation and confidence region estimation. A point estimator is a mapping $\theta^{*}: \mathbb{R}^{n} \rightarrow$ $\mathbb{R}^{d}$. Its quality is usually characterized by the risk function

$$
R\left(\theta, \theta^{*}\right)=\mathrm{E}_{\theta} l\left(\theta, \theta^{*}\right)
$$

where $l(u, v)$ is a loss function.

In this paper we deal with confidence region estimation. Let $P \in(0,1)$ be a given number called the confidence level. A confidence region estimator or, simply, a confidence region of level $P$, is defined as a mapping $B=B(x)$ : $\mathbb{R}^{n} \rightarrow \mathcal{B}^{d}$ such that for all $\theta \in \Theta$,

$$
\mathrm{P}_{\theta}(\theta \in B(x)) \geq P .
$$

We omit the details relating to the measurability of $B(x)$.

2000 Mathematics Subject Classification: 62F25, 62F12.

Key words and phrases: location-scale family, parameter estimation, asymptotic expansion. 
A confidence region $B(x)$ is called strong if for all $\theta \in \Theta$,

$$
\mathrm{P}_{\theta}(\theta \in B(x))=P .
$$

The quality of a confidence region can be characterized by the risk defined as

$$
R(\theta, B)=\mathrm{E}_{\theta} \lambda_{d}(B(x))
$$

where $\lambda_{d}$ is the Lebesgue measure on $\mathcal{B}^{d}$.

Let $\mathbf{B}$ be a family of strong confidence regions of level $P$ and assume that it is sufficiently rich. We say that a confidence region $B(x) \in \mathbf{B}$ is optimal if it minimizes the risk over $\mathbf{B}$. The question is: how can we find an optimal confidence region provided it exists?

One of the simplest cases where the problem can be successfully solved is provided by location-scale families, of the form

$$
\mathcal{F}=\left\{F_{\theta}: F_{\theta}(u)=F_{(0,1)}\left(\frac{u-\theta_{1}}{\theta_{2}}\right), u \in \mathbb{R}^{1}, \theta=\left(\theta_{1}, \theta_{2}\right) \in \mathbb{R}^{1} \times \mathbb{R}_{+}^{1}\right\},
$$

where the standard distribution function $F_{(0,1)}$ is assumed to be absolutely continuous.

In this case, a confidence region can be built on the basis of the sample mean

$$
\bar{x}=\frac{1}{n} \sum_{i=1}^{n} x_{i}
$$

and the sample variance

$$
s^{2}=\frac{1}{n} \sum_{j=1}^{n}\left(x_{j}-\bar{x}\right)^{2} .
$$

It is easily seen that for any $A \in \mathcal{B}^{2}$,

$$
\mathrm{P}_{\theta}\left(\left(\frac{\theta_{1}-\bar{x}}{s}, \frac{\theta_{2}-s}{s}\right) \in A\right)=\mathrm{P}_{(0,1)}\left(\left(-\frac{\bar{x}}{s}, \frac{1}{s}-1\right) \in A\right) .
$$

Consider the class of sets

$$
\mathcal{A}_{P}=\left\{A \in \mathcal{B}^{2}: \mathrm{P}_{(0,1)}\left(\left(-\frac{\bar{x}}{s}, \frac{1}{s}-1\right) \in A\right)=P\right\}
$$

and the corresponding class of strong confidence regions

$$
\mathbf{B}=\left\{B \in \mathcal{B}^{2}: B=B_{A}(x)=(\bar{x}, s)+s A, A \in \mathcal{A}_{P}\right\} .
$$

Obviously,

$$
\mathrm{P}_{\theta}\left(\theta \in B_{A}(x)\right)=P .
$$

The risk corresponding to $B_{A}$ has the form

$$
R\left(\theta, B_{A}\right)=\lambda_{2}(A) \mathrm{E}_{\theta} s^{2}=\theta_{2}^{2}((n-1) / n) b \lambda_{2}(A)
$$


where

$$
b=\operatorname{Var}_{(0,1)} \xi \text {. }
$$

In view of (1.2), a confidence region $B_{A^{*}}(x)$ is optimal over $\mathbf{B}$ if it has the minimal volume, that is, if

$$
A^{*} \in \arg \min _{A \in \mathcal{A}_{P}} \lambda_{2}(A) \text {. }
$$

So, we come to the problem of searching minimum volume sets (see e.g. Jeyaratnam, 1985, or Czarnowska and Nagaev, 2001). We adopt a method suggested in Czarnowska and Nagaev (2001) and based on the result from Einmahl and Mason (1992). A connection between the minimum volume confidence regions and the level sets of a density function should be emphasized (see e.g. Polonik, 1997, and Garcia et al., 2003). The case $d=1$ is discussed for example in Pratt (1961), Guenther (1969), Bartoszyński and Chan (1990).

In what follows we assume that the joint distribution of the two-dimensional statistic $(-\bar{x} / s, 1 / s-1)$, when the sample $x$ is drawn from the distribution $F_{(0,1)}$, is absolutely continuous with density $p_{n}(u)=p_{n}\left(u_{1}, u_{2}\right)$ and such that for all $z>0$,

$$
\int_{\left\{u \in \mathbb{R}^{2}: p_{n}(u)=z\right\}} p_{n}(u) d u=0 .
$$

For any $z>0$ define

$$
A_{z}=\left\{u \in \mathbb{R}^{2}: p_{n}(u) \geq z\right\}
$$

and choose $z=z_{P}$ so that

$$
\int_{A_{z_{P}}} p_{n}(u) d u=P
$$

Then

$$
A_{z_{P}} \in \mathcal{A}_{P}=\left\{A \in \mathcal{B}^{2}: \int_{A} p_{n}(u) d u=P\right\} .
$$

By Proposition 2.1 of Einmahl and Mason (1992),

$$
A_{z_{P}} \in \arg \min _{A \in \mathcal{A}_{P}} \lambda_{2}(A) \text {. }
$$

Moreover, if $A \in \arg \min _{A \in \mathcal{A}_{P}} \lambda_{2}(A)$, then

$$
\lambda_{2}\left(A \triangle A_{z_{P}}\right)=0 \text {. }
$$

Thus, the optimal confidence region $B_{\text {opt }}(x)$ over $\mathbf{B}$ has the form

$$
B_{\text {opt }}(x)=(\bar{x}, s)+s A_{z_{P}} .
$$

Recall that the class of confidence regions $\mathbf{B}$ has been built on the basis of the statistics $\bar{x}$ and $s$. Obviously, we could take for this purpose any two 
statistics $t_{1}(x)$ and $t_{2}(x)$ such that for all $\left(\theta_{1}, \theta_{2}\right) \in \mathbb{R}^{1} \times \mathbb{R}_{+}^{1}$,

$$
t_{1}\left(\theta_{2} x+\theta_{1} \mathbf{1}\right)=\theta_{2} t_{1}(x)+\theta_{1}, \quad t_{2}\left(\theta_{2} x+\theta_{1} \mathbf{1}\right)=\theta_{2} t_{2}(x),
$$

where $\mathbf{1}=(1,1, \ldots, 1)$. Observe that Czarnowska and Nagaev (2001) studied three location-scale families: normal, exponential and uniform. The statistics $t_{1}(x)$ and $t_{2}(x)$ were chosen there in the following way:

$$
t_{1}(x)= \begin{cases}\bar{x} & \text { if } F_{(0,1)} \text { is normal, } \\ x_{(1)} & \text { if } F_{(0,1)} \text { is exponential } \\ (1 / 2)\left(x_{(1)}+x_{(n)}\right) & \text { if } F_{(0,1)} \text { is uniform, }\end{cases}
$$

and

$$
t_{2}(x)= \begin{cases}s & \text { if } F_{(0,1)} \text { is normal, } \\ \bar{x}-x_{(1)} & \text { if } F_{(0,1)} \text { is exponential, } \\ x_{(n)}-x_{(1)} & \text { if } F_{(0,1)} \text { is uniform, }\end{cases}
$$

where $x_{(1)}$ and $x_{(n)}$ are the extreme left and extreme right order statistics, respectively. Note that to compare with Czarnowska and Nagaev (2001) we have exchanged $t_{1}$ and $t_{2}$ for convenience.

In the present paper we do not specify a location-scale family but suggest an asymptotic approach to the problem. This approach consists in using $\bar{x}$ and $s$ as $t_{1}(x)$ and $t_{2}(x)$, respectively, for all sufficiently large samples. We provide an asymptotic analysis of the optimal confidence region given by (1.3). This analysis is based on the asymptotic expansion of the density $p_{n}\left(u_{1}, u_{2}\right)$, as in Tonda and Wakaki (2003), where an asymptotic method for testing the equality of variances in a population was used. Despite of what was written in Czarnowska and Nagaev (2001), the asymptotic results are important since they show the tendency in changes of the optimal confidence region with increasing sample size, and they are applicable without specifying a location-scale family.

The paper is organized as follows. In Section 2 an asymptotic expansion of the density is obtained. In Section 3 we give an expansion of the confidence region given by (1.3) and construct its estimator. At the end of the section we compare the quality of both sets. The proofs are collected in Section 4 .

2. Asymptotic expansion of density. Let $Y=\left(Y_{1}, Y_{2}\right), Y^{(1)}=$ $\left(Y_{11}, Y_{12}\right), Y^{(2)}=\left(Y_{21}, Y_{22}\right), \ldots$ be independent and identically distributed two-dimensional random vectors. Assume that $\mathrm{E} Y_{1}=\mathrm{E} Y_{2}=0$ and that the covariance matrix $V$ of $Y$ is non-degenerate. Following Bhattacharya and Ranga Rao $(1976, \S 6)$ for a given two-dimensional vector $\mu=\left(\mu_{1}, \mu_{2}\right)$ with non-negative integer components we set

$$
|\mu|=\mu_{1}+\mu_{2}, \quad \mu !=\mu_{1} ! \mu_{2} !
$$


Denote by $\chi_{\mu}$ the $\mu$ th cumulant of $Y$. Under our assumptions, we have

$$
\chi_{1,0}=\chi_{0,1}=0, \quad \chi_{2,0}=\mathrm{EY}_{1}^{2}, \quad \chi_{1,1}=\mathrm{EY}_{1} Y_{2}, \quad \chi_{0,2}=\mathrm{EY}_{2}^{2} .
$$

If $\mathrm{E}|Y|^{3}<\infty$, then

$$
\chi_{3,0}=\mathrm{E} Y_{1}^{3}, \quad \chi_{2,1}=\mathrm{E} Y_{1}^{2} Y_{2}, \quad \chi_{1,2}=\mathrm{E} Y_{1} Y_{2}^{2}, \quad \chi_{0,3}=\mathrm{E} Y_{2}^{3} .
$$

But if $\mathrm{E}|Y|^{4}<\infty$, then

$$
\begin{array}{ll}
\chi_{4,0}=\mathrm{E} Y_{1}^{4}-3\left(\mathrm{E} Y_{1}^{2}\right)^{2}, & \chi_{0,4}=\mathrm{E} Y_{2}^{4}-3\left(\mathrm{E} Y_{2}^{2}\right)^{2}, \\
\chi_{3,1}=\mathrm{E} Y_{1}^{3} Y_{2}-3 \mathrm{E} Y_{1} Y_{2} \mathrm{E} Y_{1}^{2}, & \chi_{1,3}=\mathrm{E} Y_{1} Y_{2}^{3}-3 \mathrm{E} Y_{1} Y_{2} \mathrm{E} Y_{2}^{2}
\end{array}
$$

and

$$
\chi_{2,2}=\mathrm{E} Y_{1}^{2} Y_{2}^{2}-\mathrm{E} Y_{1}^{2} \mathrm{E} Y_{2}^{2}-2\left(\mathrm{E} Y_{1} Y_{2}\right)^{2} .
$$

Let the functions $P_{1}\left(-\varphi_{\mathbf{0}, V}\right)(y)$ and $P_{2}\left(-\varphi_{\mathbf{0}, V}\right)(y)$, as in Bhattacharya and Ranga Rao $(1976, \S 7)$, be defined as

$$
P_{1}\left(-\varphi_{\mathbf{0}, V}\right)(y)=-\sum_{|\mu|=3} \frac{\chi_{\mu}}{\mu !} \mathrm{D}^{\mu} \varphi_{\mathbf{0}, V}(y),
$$

$$
P_{2}\left(-\varphi_{\mathbf{0}, V}\right)(y)=\sum_{|\mu|=4} \frac{\chi_{\mu}}{\mu !} \mathrm{D}^{\mu} \varphi_{\mathbf{0}, V}(y)+\sum_{|\mu|=6} \bar{\chi}_{\mu} \mathrm{D}^{\mu} \varphi_{\mathbf{0}, V}(y)
$$

where the differential operator $\mathrm{D}^{\mu}$ is given by

$$
\mathrm{D}^{\mu} \varphi_{\mathbf{0}, V}(y)=\frac{\partial^{|\mu|}}{\partial y_{1}^{\mu_{1}} \partial y_{2}^{\mu_{2}}} \varphi_{\mathbf{0}, V}(y)
$$

and $\varphi_{\mathbf{0}, \mathrm{V}}(y)$ denotes the density of the two-dimensional normal distribution with zero mean vector and covariance matrix $V$. The coefficients $\bar{\chi}_{\mu}$ for $|\mu|=6$ are

$$
\begin{aligned}
\bar{\chi}_{6,0}=\frac{1}{72} \chi_{3,0}^{2}, & \bar{\chi}_{0,6}=\frac{1}{72} \chi_{0,3}^{2}, \\
\bar{\chi}_{5,1}=\frac{1}{12} \chi_{3,0} \chi_{2,1}, \quad \bar{\chi}_{1,5} & =\frac{1}{12} \chi_{0,3} \chi_{1,2}, \\
\bar{\chi}_{4,2}=\frac{1}{8} \chi_{2,1}^{2}+\frac{1}{12} \chi_{3,0} \chi_{1,2}, & \bar{\chi}_{2,4}=\frac{1}{8} \chi_{1,2}^{2}+\frac{1}{12} \chi_{0,3} \chi_{2,1},
\end{aligned}
$$

and

$$
\bar{\chi}_{3,3}=\frac{1}{36} \chi_{3,0} \chi_{0,3}+\frac{1}{4} \chi_{2,1} \chi_{1,2} .
$$

By direct calculations, from (2.5) and (2.6) it follows that there exist polynomials $Q_{1}(y)$ of order 3 and $Q_{2}(y)$ of order 6 such that

$$
P_{1}\left(-\varphi_{\mathbf{0}, V}\right)(y)=Q_{1}(y) \varphi_{\mathbf{0}, V}(y), \quad P_{2}\left(-\varphi_{\mathbf{0}, V}\right)(y)=Q_{2}(y) \varphi_{\mathbf{0}, V}(y) .
$$

From Bhattacharya and Ranga Rao (1976, Theorem 19.2) we derive the following statement. 
Proposition 2.1. Assume that $\mathrm{E}|Y|^{4}<\infty$. If there exists $n_{0} \geq 1$ such that the density $p_{n_{0}, Y}(y)$ corresponding to the normalized sum $n_{0}^{-1 / 2}\left(Y^{(1)}+\right.$ $\left.\cdots+Y^{\left(n_{0}\right)}\right)$ is bounded, then

$$
\sup _{y \in \mathbb{R}^{2}}\left|p_{n, Y}(y)-\varphi_{\mathbf{0}, V}(y)\left(1+n^{-1 / 2} Q_{1}(y)+n^{-1} Q_{2}(y)\right)\right|=o\left(n^{-1}\right), \quad n \rightarrow \infty,
$$

where $Q_{1}(y)$ and $Q_{2}(y)$ are defined by $(2.7)$.

Now, assume that

$$
Y \stackrel{d}{=}\left(\xi, \xi^{2}-1\right)
$$

Obviously, the distribution of $Y$ is not absolutely continuous. However, under certain additional conditions there exists $n_{0} \geq 1$ such that the $n_{0}$ th convolution of $Y$ has a bounded density. This is so, for example, if $\xi$ has a differentiable density $p(u)$ such that

$$
\int_{-\infty}^{\infty}|u| p(u) d u<\infty \text { and } \int_{-\infty}^{\infty}\left|p^{\prime}(u)\right| d u<\infty
$$

(see e.g. Sadikova, 1966).

Denote by $b_{k}$ the $k$ th moment of $\xi$ and assume that $b_{1}=0, b_{2}=1$. Then, obviously, $\mathrm{E} Y_{1}=\mathrm{E} Y_{2}=0$ while the covariance matrix of $Y$ is

$$
V=\left(\begin{array}{cc}
1 & b_{3} \\
b_{3} & b_{4}-1
\end{array}\right) \text {. }
$$

The third order cumulants are given by

$$
\chi_{3,0}=b_{3}, \quad \chi_{2,1}=b_{4}-1, \quad \chi_{1,2}=b_{5}-2 b_{3}, \quad \chi_{0,3}=b_{6}-3 b_{4}+2,
$$

while those of order 4 are

$$
\begin{aligned}
& \chi_{4,0}=b_{4}-3, \quad \chi_{3,1}=b_{5}-4 b_{3}, \quad \chi_{2,2}=b_{6}-3 b_{4}-2 b_{3}^{2}+2, \\
& \chi_{1,3}=b_{7}-3 b_{5}+6 b_{3}-3 b_{3} b_{4}, \quad \chi_{0,4}=b_{8}-4 b_{6}+12 b_{4}-3 b_{4}^{2}-6
\end{aligned}
$$

provided $b_{8}$ exists.

Let $x_{1}, \ldots, x_{n}$ be independent copies of $\xi$. Let, as before, $\bar{x}$ and $s^{2}$ be respectively sample mean and sample variance corresponding to the sample $x=\left(x_{1}, \ldots, x_{n}\right)$.

With the help of Proposition 2.1, the local limit theorem for the density of the statistic

$$
T=T(x)=\sqrt{n}\left(-\frac{\bar{x}}{s}, \frac{1}{s}-1\right)
$$

can be established. 
Introduce the following notations:

$$
\begin{aligned}
& \mathbf{h}^{(0)}(t)=\left(-t_{1},-2 t_{2}\right), \\
& \mathbf{h}^{(1)}(t)=\left(t_{1} t_{2}, t_{1}^{2}+3 t_{2}^{2}\right), \\
& \mathbf{h}^{(2)}(t)=\left(-t_{1} t_{2}^{2},-2 t_{1}^{2} t_{2}-4 t_{2}^{3}\right)
\end{aligned}
$$

and

$$
\begin{aligned}
& A_{1}(t)=-\mathbf{h}^{(0)}(t) V^{-1}\left(\mathbf{h}^{(1)}(t)\right)^{T}, \\
& A_{2}(t)=-\mathbf{h}^{(0)}(t) V^{-1}\left(\mathbf{h}^{(2)}(t)\right)^{T}-\frac{1}{2} \mathbf{h}^{(1)}(t) V^{-1}\left(\mathbf{h}^{(1)}(t)\right)^{T} .
\end{aligned}
$$

Let $\langle\cdot, \cdot\rangle$ be an inner product in $\mathbb{R}^{2}$.

LEMma 2.2. Assume that $\mathrm{E} \xi^{8}<\infty$ and that the density of $\xi$ satisfies (2.8). Then for any $R>0$ the density $p_{n, T}(t)$ of the statistic $T$ given by (2.10) satisfies

$$
\sup _{|t| \leq R}\left|p_{n, T}(t)-\varphi_{\mathbf{0}, \mathrm{W}}(t)\left(1+n^{-1 / 2} P_{1}(t)+n^{-1} P_{2}(t)\right)\right|=o\left(n^{-1}\right), \quad n \rightarrow \infty
$$

where

$$
W=\left(\begin{array}{cc}
1 & b_{3} / 2 \\
b_{3} / 2 & \left(b_{4}-1\right) / 4
\end{array}\right)
$$

while the polynomials $P_{1}(t)$ of order 3 and $P_{2}(t)$ of order 6 are given by

$$
\begin{aligned}
P_{1}(t)= & -4 t_{2}+A_{1}(t)+Q_{1}\left(\mathbf{h}^{(0)}(t)\right), \\
P_{2}(t)= & -6 t_{2}^{2}-4 t_{2} P_{1}(t)+A_{1}(t) Q_{1}\left(\mathbf{h}^{(0)}(t)\right)+A_{2}(t) \\
& +\frac{1}{2} A_{1}^{2}(t)+Q_{2}\left(\mathbf{h}^{(0)}(t)\right)+\left\langle\gamma(t), \mathbf{h}^{(1)}(t)\right\rangle
\end{aligned}
$$

where

$$
\gamma(t)=\operatorname{grad} Q_{1}\left(\mathbf{h}^{(0)}(t)\right)
$$

3. Main results. Recall that the main objective of the paper is to study the asymptotic properties of the confidence region given by (1.3). More precisely, we suggest something like an asymptotic expansion of the region $A_{n}=A_{z_{P}^{(n)}}$. To simplify our notation, we write $z_{P}$ instead of $z_{P}^{(n)}$.

Observe that

$$
A_{n}=\left\{u \in \mathbb{R}^{2}: p_{n}(u) \geq z_{P}\right\}=\frac{1}{\sqrt{n}}\left\{t \in \mathbb{R}^{2}: p_{n, T}(t) \geq t_{P}\right\}
$$

where the density $p_{n, T}(t)$ corresponds to $T$ given by $(2.10)$ while $t_{P}=z_{P} / n$ satisfies the condition

$$
\int_{\left\{t: p_{n, T}(t) \geq t_{P}\right\}} p_{n, T}(t) d t=P .
$$


Recall that any point $e \in S^{1}$, where $S^{1}$ is the unit sphere in $\mathbb{R}^{2}$, can be written as

$$
e=\left(e_{1}, e_{2}\right)=(\cos \alpha, \sin \alpha), \quad \alpha \in[0,2 \pi) .
$$

Using the asymptotic expansion for $p_{n, T}(t)$ given by Lemma 2.2, we obtain the asymptotic expansion for $A_{n}$.

Theorem 3.1. Assume that $\mathrm{E} \xi^{8}<\infty$ and that the density of $\xi$ satisfies (2.8). Then

$$
\begin{array}{r}
A_{n}=\frac{1}{\sqrt{n}}\left\{(r, \alpha): 0 \leq r \leq r_{0}(\alpha)\left[1+n^{-1 / 2} R_{1}(\alpha)+n^{-1} R_{2}(\alpha)+o\left(n^{-1}\right)\right],\right. \\
\alpha \in[0,2 \pi)\}
\end{array}
$$

where

$$
\begin{aligned}
& r_{0}=r_{0}(\alpha)=\frac{l^{1 / 2}}{\sigma(\alpha)}, \quad l=-2 \ln (1-P), \\
& \sigma^{2}(\alpha)=(\cos \alpha, \sin \alpha) W^{-1}(\cos \alpha, \sin \alpha)^{T}, \\
& R_{1}(\alpha)=\frac{H_{1}\left(r_{0}, \alpha\right)}{l}, \\
& R_{2}(\alpha)=\frac{H_{1}^{\prime}\left(r_{0}, \alpha\right) H_{1}\left(r_{0}, \alpha\right)}{l^{3 / 2} \sigma(\alpha)}+\frac{H_{2}\left(r_{0}, \alpha\right)}{l} \\
& \quad-\frac{H_{1}^{2}\left(r_{0}, \alpha\right)}{2 l}(1 / l+1)-\frac{B+C+D}{2 \pi l \sqrt{\operatorname{det} W}}
\end{aligned}
$$

and

$$
\begin{aligned}
H_{i}(r, \alpha) & =P_{i}(r \cos \alpha, r \sin \alpha), i=1,2, \quad H_{1}^{\prime}\left(r_{0}(\alpha), \alpha\right)=\left.\frac{\partial}{\partial r} H_{1}(r, \alpha)\right|_{r=r_{0}}, \\
B & =\int_{0}^{2 \pi} \frac{H_{1}^{\prime}\left(r_{0}(\beta), \beta\right) H_{1}\left(r_{0}(\beta), \beta\right)}{l^{1 / 2} \sigma^{3}(\beta)} d \beta, \quad C=\int_{0}^{2 \pi} \frac{H_{2}\left(r_{0}(\beta), \beta\right)}{\sigma^{2}(\beta)} d \beta, \\
D & =\frac{1}{1-P} \int_{0}^{2 \pi} \int_{0}^{r_{0}(\beta)} r e^{-r^{2} \sigma^{2}(\beta) / 2} H_{2}(r, \beta) d r d \beta .
\end{aligned}
$$

Since for large $n$ the density $p_{n, T}(t)$ can be approximated by $\varphi_{0, W}(t)$, let us consider the set $A_{n, 0}$ satisfying the condition

$$
\int_{\sqrt{n} A_{n, 0}} p_{n, T}(t) d t=\int_{\left\{t: \varphi_{0, W}(t) \geq \bar{t}_{P}\right\}} p_{n, T}(t) d t=P
$$

where $\bar{t}_{P}$ is a constant depending on $\xi, n$ and $P$.

The following theorem gives the asymptotic expansion of $A_{n, 0}$.

TheOREM 3.2. Suppose that the conditions of Theorem 3.1 are satisfied. 
Then

$$
A_{n, 0}=\frac{1}{\sqrt{n}}\left\{(r, \alpha): 0 \leq r \leq r_{0}(\alpha)\left[1+n^{-1} \bar{R}_{2}+o\left(n^{-1}\right)\right], \alpha \in[0,2 \pi)\right\}
$$

where

$$
\bar{R}_{2}=-\frac{D}{2 \pi l \sqrt{\operatorname{det} W}} .
$$

So, we have two confidence regions:

$$
B_{n}(x)=(\bar{x}, s)+s A_{n} \quad \text { and } \quad B_{n, 0}(x)=(\bar{x}, s)+s A_{n, 0} .
$$

Obviously, the volume of $A_{n}$ is smaller than that of $A_{n, 0}$, though the latter is more convenient.

The question is: how good is the approximating confidence region $B_{n, 0}(x)$ ? Or in other words, how well does $B_{n, 0}(x)$ approximate $B_{n}(x)$ ? Since $R\left(\theta, B_{n}\right) \rightarrow 0$ and $R\left(\theta, B_{n, 0}\right) \rightarrow 0$ as $n \rightarrow \infty$, it is justified to compare their ratio. In view of (1.2),

$$
\frac{R\left(\theta, B_{n}\right)}{R\left(\theta, B_{n, 0}\right)}=\frac{\lambda_{2}\left(A_{n}\right)}{\lambda_{2}\left(A_{n, 0}\right)} .
$$

THEOREM 3.3. Suppose that the conditions of Theorem 3.1 are satisfied. Let $B_{n}(x)$ and $B_{n, 0}(x)$ be given by (3.18). Then

$$
\lim _{n \rightarrow \infty} n\left(\frac{R\left(\theta, B_{n, 0}\right)}{R\left(\theta, B_{n}\right)}-1\right)=K_{P}
$$

where

$$
K_{P}=\frac{1}{2 \pi l \sqrt{\operatorname{det} W}} \int_{0}^{2 \pi} \frac{H_{1}^{2}\left(r_{0}, \alpha\right)}{\sigma^{2}(\alpha)} d \alpha .
$$

Corollary 3.4. If in addition to the conditions of Theorem 3.1, the distribution of $\xi$ is symmetric, then

$$
K_{P}=K_{1}+l K_{2}+l^{2} K_{3}
$$

where

$$
\begin{aligned}
& K_{1}=\frac{\left(3 b_{4}^{2}-3 b_{4}+1-b_{6}\right)^{2}}{8\left(b_{4}-1\right)^{3}}, \\
& K_{2}=\frac{\left(b_{6}-3 b_{4}^{2}+3 b_{4}-1\right)\left(-8 b_{4}+1-2 b_{6}+9 b_{4}^{2}\right)}{32\left(b_{4}-1\right)^{3}}, \\
& K_{3}=\frac{810 b_{4}^{2}-480 b_{4}+149-864 b_{4}^{3}+192 b_{6} b_{4}-52 b_{6}+20 b_{6}^{2}-180 b_{6} b_{4}^{2}+405 b_{4}^{4}}{2304\left(b_{4}-1\right)^{3}} .
\end{aligned}
$$

EXAMPLE 3.5. Let $\xi$ have the normal distribution with density

$$
p_{(a, \sigma)}(u)=\frac{1}{\sqrt{2 \pi} \sigma} \exp \left(-\frac{(u-a)^{2}}{2 \sigma^{2}}\right), \quad u \in \mathbb{R}^{1} .
$$


Then $b_{1}=0$ and $b_{2}=1$ provided that $a=0$ and $\sigma=1$. The higher moments are $b_{4}=3, b_{6}=15$ and $b_{8}=105$. The odd moments are zero by symmetry of the density. Then

$$
K_{P}=\frac{1}{4}+\frac{7}{8} \ln (1-P)+\frac{221}{288} \ln ^{2}(1-P)
$$

and the polynomials $P_{1}$ and $P_{2}$ are given by

$$
\begin{aligned}
& P_{1}(t)=-t_{2}+t_{1}^{2} t_{2}+\frac{5}{3} t_{2}^{3} \\
& P_{2}(t)=t_{2}^{2}-\frac{5}{2} t_{1}^{2} t_{2}^{2}-\frac{47}{12} t_{2}^{4}+\frac{1}{2} t_{1}^{4} t_{2}^{2}+\frac{5}{3} t_{1}^{2} t_{2}^{4}+\frac{25}{18} t_{2}^{6}-\frac{11}{12} .
\end{aligned}
$$

EXAMPLE 3.6. Let $\xi$ have the Laplace distribution with density

$$
p_{(a, \sigma)}(u)=\frac{1}{2 \sigma} \exp \left(-\frac{|u-a|}{\sigma}\right), \quad u \in \mathbb{R}^{1} .
$$

The conditions $b_{1}=0$ and $b_{2}=1$ are satisfied if $a=0$ and $\sigma=1 / \sqrt{2}$. Then $b_{2 k-1}=0$ and $b_{2 k}=(2 k) ! / 2^{k}$ for $k=1,2, \ldots$ Therefore,

$$
K_{P}=\frac{1}{1000}+\frac{97}{2000} \ln (1-P)+\frac{8497}{14400} \ln ^{2}(1-P) .
$$

In this case,

$$
\begin{aligned}
P_{1}(t)= & -\frac{1}{25} t_{2}+\frac{2}{5} t_{1}^{2} t_{2}+\frac{154}{375} t_{2}^{3} \\
P_{2}(t)= & -\frac{59}{25} t_{2}^{2}-\frac{29}{125} t_{1}^{2} t_{2}^{2}-\frac{787}{3750} t_{2}^{4}+\frac{2}{25} t_{1}^{4} t_{2}^{2}+\frac{308}{1875} t_{1}^{2} t_{2}^{4} \\
& +\frac{11858}{140625} t_{2}^{6}-\frac{1}{10} t_{1}^{4}+\frac{51}{50} t_{1}^{2}-\frac{11}{300} .
\end{aligned}
$$

EXAMPLE 3.7. Let $\xi$ have the Gumbel distribution with density

$$
p_{(a, \sigma)}(u)=\frac{1}{\sigma} \exp \left(-\frac{u-a}{\sigma}-\exp \left(-\frac{u-a}{\sigma}\right)\right), \quad u \in \mathbb{R}^{1} .
$$

Then $b_{1}=0$ and $b_{2}=1$ provided that

$$
a=-\frac{\sqrt{6}}{\pi} \gamma, \quad \sigma=\frac{\sqrt{6}}{\pi}
$$

where $\gamma$ is the Euler constant.

The higher moments of this distribution, calculated by Maple, are

$$
\begin{aligned}
& b_{3} \approx 1.139547100, \quad b_{4} \approx 5.400000003, \quad b_{5} \approx 18.56661600, \\
& b_{6} \approx 91.41424742, \quad b_{7} \approx 493.1498922, \quad b_{8} \approx 3091.022948 .
\end{aligned}
$$

Therefore,

$$
K_{P} \approx 0.3835836761-1.145403190 \ln (1-P)-0.6363182720 \ln ^{2}(1-P) .
$$


The polynomials in Lemma 2.2 have the forms

$$
\begin{aligned}
P_{1}(t)= & 0.845339128 t_{2}+1.287265118 t_{1}^{2} t_{2}-0.620560754 t_{1} t_{2}^{2} \\
& -0.2042000243 t_{1}^{3}+0.281444521 t_{2}^{3}-0.9432770660 t_{1}, \\
P_{2}(t)= & 3.327714146-2.570311970 t_{1}^{2}-10.48951112 t_{2}^{2}+11.26564457 t_{1} t_{2} \\
& -1.788320216 t_{1}^{3} t_{2}+2.746725415 t_{1}^{2} t_{2}^{2}-4.011241936 t_{1} t_{2}^{3} \\
& +0.2625879127 t_{1}^{4}+1.882364959 t_{2}^{4}-0.2628595872 t_{1}^{5} t_{2} \\
& +0.955244250 t_{1}^{4} t_{2}^{2}-0.856297195 t_{1}^{3} t_{2}^{3}+0.554841540 t_{1}^{2} t_{2}^{4} \\
& -0.174653399 t_{1} t_{2}^{5}+0.02084882577 t_{1}^{6}+0.039605515 t_{2}^{6} .
\end{aligned}
$$

\section{Proofs}

4.1. Proof of Lemma 2.2. It is evident that

$$
n^{-1 / 2}\left(Y^{(1)}+\cdots+Y^{(n)}\right) \stackrel{d}{=} \sqrt{n}\left(\bar{x}, s^{2}+\bar{x}^{2}-1\right) .
$$

Consider the transformation

$$
\left(\sqrt{n} \bar{x}, \sqrt{n}\left(s^{2}+\bar{x}^{2}-1\right)\right)=\left(g_{n, 1}\left(T_{1}(x), T_{2}(x)\right), g_{n, 2}\left(T_{1}(x), T_{2}(x)\right)\right)
$$

where

$$
\left\{\begin{aligned}
g_{n, 1}\left(t_{1}, t_{2}\right) & =\frac{-t_{1}}{1+n^{-1 / 2} t_{2}}, \\
g_{n, 2}\left(t_{1}, t_{2}\right) & =\sqrt{n}\left(\frac{1+n^{-1} t_{1}^{2}}{\left(1+n^{-1 / 2} t_{2}\right)^{2}}-1\right) .
\end{aligned}\right.
$$

Define

$$
\mathbf{g}_{n}(t)=\left(g_{n, 1}(t), g_{n, 2}(t)\right) .
$$

By Proposition 2.1, we have

$$
\begin{aligned}
& p_{n, T}(t)=\left|\mathbf{J}_{n}(t)\right| p_{n, Y}\left(\mathbf{g}_{n}(t)\right) \\
& =\left|\mathbf{J}_{n}(t)\right| \varphi_{\mathbf{0}, V}\left(\mathbf{g}_{n}(t)\right)\left(1+n^{-1 / 2} Q_{1}\left(\mathbf{g}_{n}(t)\right)+n^{-1} Q_{2}\left(\mathbf{g}_{n}(t)\right)\right)+o\left(n^{-1}\right)
\end{aligned}
$$

where $\mathbf{J}_{n}(t)$ is the Jacobian of the transformation $\mathbf{g}_{n}(t)$. By Taylor expansion, it admits the representation

$$
\mathbf{J}_{n}(t)=\frac{2}{\left(1+n^{-1 / 2} t_{2}\right)^{4}}=2\left(1-n^{-1 / 2} 4 t_{2}+n^{-1} 10 t_{2}^{2}\right)+o\left(n^{-1}\right)
$$

uniformly in $|t| \leq R$ where $R>0$ is any large number.

Also by Taylor expansion,

$$
\mathbf{g}_{n}(t)=\mathbf{h}^{(0)}(t)+n^{-1 / 2} \mathbf{h}^{(1)}(t)+n^{-1} \mathbf{h}^{(2)}(t)+o\left(n^{-1}\right) \mathbf{1}
$$

uniformly in $|t| \leq R$. Here, $\mathbf{1}=(1,1)$ while $\mathbf{h}^{(i)}(t), i=0,1,2$, are given by 
(2.11). Further,

$$
\begin{aligned}
\varphi_{\mathbf{0}, V}\left(\mathbf{g}_{n}(t)\right)= & \frac{1}{2 \pi}(\operatorname{det} V)^{-1 / 2} \exp \left(-\frac{1}{2} t W^{-1} t^{T}+n^{-1 / 2} A_{1}(t)+n^{-1} A_{2}(t)\right) \\
& +o\left(n^{-1}\right)
\end{aligned}
$$

uniformly in $|t| \leq R$, where $A_{1}(t)$ and $A_{2}(t)$ are given by (2.12).

Again by Taylor expansion, one can write

$$
\begin{aligned}
& Q_{1}\left(\mathbf{g}_{n}(t)\right)=Q_{1}\left(\mathbf{h}^{(0)}(t)\right)+n^{-1 / 2}\left\langle\gamma^{(1)}(t), \mathbf{h}^{(1)}(t)\right\rangle+O\left(n^{-1}\right), \\
& Q_{2}\left(\mathbf{g}_{n}(t)\right)=Q_{2}\left(\mathbf{h}^{(0)}(t)\right)+O\left(n^{-1 / 2}\right)
\end{aligned}
$$

uniformly in $|t| \leq R$.

Combining (4.19)-(4.21) we arrive at the desired statement, since $2(\operatorname{det} V)^{-1 / 2}=(\operatorname{det} W)^{-1 / 2}$.

4.2. Proof of Theorem 3.1. Using the asymptotic expansion of $p_{n, T}(t)$ given by Lemma 2.2, we rewrite (3.14) for sufficiently large $n$ as

$$
\int_{A} \varphi_{\mathbf{0}, W}(t)\left(1+\frac{1}{\sqrt{n}} P_{1}(t)+\frac{1}{n} P_{2}(t)+o\left(n^{-1}\right)\right) d t=P
$$

where

$$
A=\left\{t \in \mathbb{R}^{2}: \varphi_{\mathbf{0}, W}(t)\left(1+\frac{1}{\sqrt{n}} P_{1}(t)+\frac{1}{n} P_{2}(t)+o\left(n^{-1}\right)\right) \geq t_{P}\right\} .
$$

Represent

$$
t_{P}=z_{0}+\frac{z_{1}}{\sqrt{n}}+\frac{z_{2}}{n}+o\left(n^{-1}\right)
$$

for some constants $z_{0}, z_{1}$ and $z_{2}$.

Observe that, as $n \rightarrow \infty$,

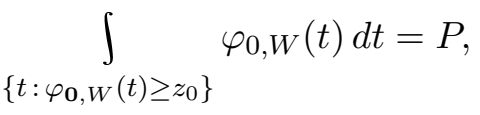

so one can calculate that

$$
z_{0}=\frac{1-P}{2 \pi \sqrt{\operatorname{det} W}}
$$

Consider the polar coordinates $\left(t_{1}, t_{2}\right)=(r \cos \alpha, r \sin \alpha)$, for $r>0$ and $\alpha \in[0,2 \pi)$. Then $|J|=r$ and

$$
t W^{-1} t^{T}=r^{2} \sigma^{2}(\alpha) .
$$

From (4.22) it follows that

$$
\begin{aligned}
\frac{1}{2 \pi \sqrt{\operatorname{det} W}} \int_{A} r e^{-r^{2} \sigma^{2}(\alpha) / 2}(1 & +\frac{1}{\sqrt{n}} H_{1}(r, \alpha) \\
& \left.+\frac{1}{n} H_{2}(r, \alpha)+o\left(n^{-1}\right)\right) d r d \alpha=P
\end{aligned}
$$


where

$$
\begin{aligned}
A=\left\{(r, \alpha): \frac{1}{2 \pi \sqrt{\operatorname{det} W}} e^{-r^{2} \sigma^{2}(\alpha) / 2}(1+\right. & \left.\frac{1}{\sqrt{n}} H_{1}(r, \alpha)+\frac{1}{n} H_{2}(r, \alpha)\right) \\
& \left.\geq z_{0}+\frac{z_{1}}{\sqrt{n}}+\frac{z_{2}}{n}+o\left(n^{-1}\right)\right\} .
\end{aligned}
$$

By (4.23) and the useful expansion

$$
\ln \left(1+\frac{1}{\sqrt{n}} a+\frac{1}{n} b\right)=\frac{1}{\sqrt{n}} a+\frac{1}{n}\left(b-\frac{1}{2} a^{2}\right)+O\left(n^{-3 / 2}\right), \quad a, b \in \mathbb{R}^{1},
$$

we obtain the equivalent form of the set $A$ for all sufficiently large $n$ :

$$
\begin{array}{r}
A=\left\{(r, \alpha): \frac{1}{2} r^{2} \sigma^{2}(\alpha)-\frac{1}{\sqrt{n}} H_{1}(r, \alpha)-\frac{1}{n}\left(H_{2}(r, \alpha)-\frac{1}{2} H_{1}^{2}(r, \alpha)\right)\right. \\
\left.\leq-\ln (1-P)-\frac{z_{1}}{\sqrt{n} z_{0}}-\frac{1}{n}\left(\frac{z_{2}}{z_{0}}-\frac{z_{1}^{2}}{2 z_{0}^{2}}\right)+o\left(n^{-1}\right)\right\} .
\end{array}
$$

Let us parametrise the boundary of the set $A$. For any $\alpha \in[0,2 \pi)$ and for all sufficiently large $n$ we try to find the following representation of the length of the radius of $A$ :

$$
r_{n}(\alpha)=r_{0}(\alpha)+\frac{r_{1}(\alpha)}{\sqrt{n}}+\frac{r_{2}(\alpha)}{n}+O\left(n^{-3 / 2}\right) .
$$

Obviously, $r_{0}(\alpha)$ satisfies the condition

$$
\frac{1}{2} r_{0}^{2}(\alpha) \sigma^{2}(\alpha)=-\ln (1-P) .
$$

Hence,

$$
r_{0}^{2}(\alpha)=\frac{-2 \ln (1-P)}{\sigma^{2}(\alpha)}=\frac{l}{\sigma^{2}(\alpha)}
$$

Clearly, we have

$$
\begin{aligned}
\frac{1}{2} r_{n}^{2}(\alpha) \sigma^{2}(\alpha)= & \frac{1}{2} \sigma^{2}(\alpha) r_{0}^{2}(\alpha)+\frac{1}{\sqrt{n}} \sigma^{2}(\alpha) r_{0}(\alpha) r_{1}(\alpha) \\
& +\frac{1}{2 n} \sigma^{2}(\alpha)\left(r_{1}^{2}(\alpha)+2 r_{0}(\alpha) r_{2}(\alpha)\right)+O\left(n^{-3 / 2}\right) \\
H_{1}\left(r_{n}(\alpha), \alpha\right)= & H_{1}\left(r_{0}, \alpha\right)+\frac{1}{\sqrt{n}} H_{1}^{\prime}\left(r_{0}, \alpha\right) r_{1}(\alpha)+O\left(n^{-1}\right) \\
H_{2}\left(r_{n}(\alpha), \alpha\right)- & \frac{1}{2} H_{1}^{2}\left(r_{n}(\alpha), \alpha\right) \\
= & H_{2}\left(r_{0}, \alpha\right)-\frac{1}{2} H_{1}^{2}\left(r_{0}, \alpha\right)+O\left(n^{-1 / 2}\right)
\end{aligned}
$$


Combining (4.27)-(4.29) and (4.25) we obtain

$$
\left\{\begin{aligned}
-\frac{z_{1}}{z_{0}}= & \sigma^{2}(\alpha) r_{1}(\alpha) r_{0}(\alpha)-H_{1}\left(r_{0}, \alpha\right) \\
-\frac{z_{2}}{z_{0}}+\frac{z_{1}^{2}}{2 z_{0}^{2}}= & \frac{1}{2} \sigma^{2}(\alpha)\left(r_{1}^{2}(\alpha)+2 r_{2}(\alpha) r_{0}(\alpha)\right)-H_{1}^{\prime}\left(r_{0}, \alpha\right) r_{1}(\alpha) \\
& -H_{2}\left(r_{0}, \alpha\right)+\frac{1}{2} H_{1}^{2}\left(r_{0}, \alpha\right)
\end{aligned}\right.
$$

Let

$$
c=\frac{1}{\sqrt{\operatorname{det} W}} .
$$

Then (4.24) has the form

$$
\begin{aligned}
\frac{c}{2 \pi} \int_{0}^{2 \pi} \int_{0}^{r_{n}(\alpha)} r e^{-r^{2} \sigma^{2}(\alpha) / 2}(1+ & \frac{1}{\sqrt{n}} H_{1}(r, \alpha) \\
& \left.+\frac{1}{n} H_{2}(r, \alpha)+o\left(n^{-1}\right)\right) d r d \alpha=P .
\end{aligned}
$$

By (4.26), for all sufficiently large $n$ we obtain

$$
\begin{aligned}
\int_{0}^{r_{n}(\alpha)} r e^{-r^{2} \sigma^{2}(\alpha) / 2}\left(1+\frac{1}{\sqrt{n}} H_{1}(r, \alpha)+\frac{1}{n} H_{2}(r, \alpha)+o\left(n^{-1}\right)\right) d r \\
=\int_{0}^{r_{0}(\alpha)} r e^{-r^{2} \sigma^{2}(\alpha) / 2}\left(1+\frac{1}{\sqrt{n}} H_{1}(r, \alpha)+\frac{1}{n} H_{2}(r, \alpha)+o\left(n^{-1}\right)\right) d r \\
+r_{0}(\alpha) e^{-r_{0}^{2}(\alpha) \sigma^{2}(\alpha) / 2}\left(1+\frac{1}{\sqrt{n}} H_{1}\left(r_{0}, \alpha\right)+\frac{1}{n} H_{2}\left(r_{0}, \alpha\right)+o\left(n^{-1}\right)\right) \\
\quad \times\left(\frac{r_{1}(\alpha)}{\sqrt{n}}+\frac{r_{2}(\alpha)}{n}+o\left(n^{-1}\right)\right) \\
\quad+\frac{1}{2}\left(1-r_{0}^{2}(\alpha) \sigma^{2}(\alpha)\right) e^{-r_{0}^{2}(\alpha) \sigma^{2}(\alpha) / 2} \frac{r_{1}^{2}(\alpha)}{n}+o\left(n^{-1}\right) .
\end{aligned}
$$

Then (4.31) yields

$$
I_{0}+\frac{1}{\sqrt{n}} I_{1}+\frac{1}{n} I_{2}+o\left(n^{-1}\right)=P
$$

where

$$
I_{0}=\frac{c}{2 \pi} \int_{0}^{2 \pi} \int_{0}^{r_{0}(\alpha)} r e^{-r^{2} \sigma^{2}(\alpha) / 2} d r d \alpha
$$




$$
\begin{aligned}
I_{1}= & \frac{c}{2 \pi} \int_{0}^{2 \pi} \int_{0}^{r_{0}(\alpha)} r e^{-r^{2} \sigma^{2}(\alpha) / 2} H_{1}(r, \alpha) d r d \alpha \\
& +\frac{c(1-P)}{2 \pi} \int_{0}^{2 \pi} r_{0}(\alpha) r_{1}(\alpha) d \alpha \\
I_{2}= & \frac{c}{2 \pi} \int_{0}^{2 \pi} \int_{0}^{r_{0}(\alpha)} r e^{-r^{2} \sigma^{2}(\alpha) / 2} H_{2}(r, \alpha) d r d \alpha \\
& +\frac{c(1-P)}{2 \pi} \int_{0}^{2 \pi} r_{0}(\alpha)\left(r_{2}(\alpha)+H_{1}\left(r_{0}, \alpha\right) r_{1}(\alpha)\right) d \alpha \\
& +\frac{c(1-P)(1-l)}{4 \pi} \int_{0}^{2 \pi} r_{1}^{2}(\alpha) d \alpha .
\end{aligned}
$$

By direct calculations, one can show that $I_{0}=P$ and

$$
\frac{c}{2 \pi} \int_{0}^{2 \pi} \frac{1}{\sigma^{2}(\alpha)} d \alpha=1
$$

Therefore, $I_{1}=I_{2}=0$. The formulas for $r_{1}(\alpha)$ and $r_{2}(\alpha)$ can then be found from (4.33) and (4.34).

Let us represent $I_{1}=(c / 2 \pi) I_{11}+I_{12}$, where

$$
I_{11}=\int_{0}^{2 \pi} \int_{0}^{r_{0}(\alpha)} r e^{-r^{2} \sigma^{2}(\alpha) / 2} H_{1}(r, \alpha) d r d \alpha, \quad I_{12}=\frac{c(1-P)}{2 \pi} \int_{0}^{2 \pi} r_{0}(\alpha) r_{1}(\alpha) d \alpha .
$$

Changing variables, we have

$$
\begin{aligned}
I_{11} & =\int_{0}^{2 \pi} \int_{0}^{l^{1 / 2}} \frac{t}{\sigma^{2}(\alpha)} e^{-t^{2} / 2} H_{1}\left(\frac{t}{\sigma(\alpha)}, \alpha\right) d t d \alpha \\
& =\int_{0}^{l^{1 / 2}} t^{4} e^{-t^{2} / 2} d t \int_{0}^{2 \pi} \frac{L_{3}(\alpha)}{\sigma^{5}(\alpha)} d \alpha+\int_{0}^{l^{1 / 2}} t^{2} e^{-t^{2} / 2} d t \int_{0}^{2 \pi} \frac{L_{1}(\alpha)}{\sigma^{3}(\alpha)} d \alpha=0,
\end{aligned}
$$

since $\sigma(\alpha)$ is an even function and $H_{1}(r, \alpha)=L_{3}(\alpha) r^{3}+L_{1}(\alpha) r$, where $L_{i}(\alpha)=L_{i}(\cos \alpha, \sin \alpha), i=1,3$, are homogeneous polynomials of degree $i$.

For $I_{12}$, by (4.30) and (4.35), it follows that

$$
I_{12}=\frac{c(1-P)}{2 \pi} \int_{0}^{2 \pi}\left(\frac{H_{1}\left(r_{0}, \alpha\right)-z_{1} / z_{0}}{\sigma^{2}(\alpha)}\right) d \alpha=\frac{-(1-P) z_{1}}{z_{0}} .
$$


Here, we use the identity

$$
\int_{0}^{2 \pi} \frac{H_{1}\left(r_{0}, \alpha\right)}{\sigma^{2}(\alpha)} d \alpha=0,
$$

which holds due to the same arguments as for (4.36). Hence,

$$
I_{1}=-(1-P) \frac{z_{1}}{z_{0}}
$$

that is,

$$
z_{1}=0
$$

Then, by (4.30),

$$
r_{1}(\alpha)=\frac{H_{1}\left(r_{0}, \alpha\right)}{\sigma^{2}(\alpha) r_{0}(\alpha)}=r_{0}(\alpha) R_{1}(\alpha) .
$$

Similarly, let us represent $I_{2}=(c / 2 \pi) I_{21}+I_{22}$, where

$$
\begin{aligned}
I_{21} & =\int_{0}^{2 \pi} \int_{0}^{r_{0}(\alpha)} r e^{-r^{2} \sigma^{2}(\alpha) / 2} H_{2}(r, \alpha) d r d \alpha \\
I_{22} & =\frac{c(1-P)}{2 \pi} \int_{0}^{2 \pi}\left[r_{0}(\alpha)\left(r_{2}(\alpha)+H_{1}\left(r_{0}, \alpha\right) r_{1}(\alpha)\right)+\frac{1-l}{2} r_{1}^{2}(\alpha)\right] d \alpha .
\end{aligned}
$$

By (4.38), we have

$$
\begin{aligned}
r_{0}(\alpha)\left(r_{2}(\alpha)+H_{1}\left(r_{0}, \alpha\right) r_{1}(\alpha)\right) & +\frac{1-l}{2} r_{1}^{2}(\alpha) \\
= & r_{0}(\alpha) r_{2}(\alpha)+\frac{H_{1}^{2}\left(r_{0}, \alpha\right)}{2 \sigma^{2}(\alpha)}\left(1+\frac{1}{l}\right) .
\end{aligned}
$$

From (4.30), (4.37) and (4.38) it follows that

$$
\begin{aligned}
-\frac{z_{2}}{z_{0}}= & \frac{1}{2} \sigma^{2}(\alpha)\left(\frac{H_{1}^{2}\left(r_{0}, \alpha\right)}{\sigma^{4}(\alpha) r_{0}^{2}(\alpha)}+2 r_{2}(\alpha) r_{0}(\alpha)\right)-\frac{H_{1}^{\prime}\left(r_{0}, \alpha\right) H_{1}\left(r_{0}, \alpha\right)}{\sigma^{2}(\alpha) r_{0}(\alpha)} \\
& -H_{2}\left(r_{0}, \alpha\right)+\frac{1}{2} H_{1}^{2}\left(r_{0}, \alpha\right) \\
= & \frac{H_{1}^{2}\left(r_{0}, \alpha\right)}{2 l}+\sigma^{2}(\alpha) r_{2}(\alpha) r_{0}(\alpha)-\frac{H_{1}^{\prime}\left(r_{0}, \alpha\right) H_{1}\left(r_{0}, \alpha\right)}{\sigma^{2}(\alpha) r_{0}(\alpha)} \\
& -H_{2}\left(r_{0}, \alpha\right)+\frac{1}{2} H_{1}^{2}\left(r_{0}, \alpha\right) .
\end{aligned}
$$

Hence

$$
\begin{aligned}
r_{2}(\alpha) r_{0}(\alpha)= & -\frac{z_{2}}{z_{0} \sigma^{2}(\alpha)}+\frac{H_{1}^{\prime}\left(r_{0}, \alpha\right) H_{1}\left(r_{0}, \alpha\right)}{\sigma^{4}(\alpha) r_{0}(\alpha)} \\
& +\frac{H_{2}\left(r_{0}, \alpha\right)}{\sigma^{2}(\alpha)}-\frac{H_{1}^{2}\left(r_{0}, \alpha\right)}{2 \sigma^{2}(\alpha)}\left(1+\frac{1}{l}\right)
\end{aligned}
$$


and (4.39) has the form

$$
\begin{aligned}
r_{0}(\alpha) r_{2}(\alpha)+\frac{H_{1}^{2}\left(r_{0}, \alpha\right)}{2 \sigma^{2}(\alpha)}\left(1+\frac{1}{l}\right)= & -\frac{z_{2}}{z_{0} \sigma^{2}(\alpha)}+\frac{H_{1}^{\prime}\left(r_{0}, \alpha\right) H_{1}\left(r_{0}, \alpha\right)}{\sigma^{4}(\alpha) r_{0}(\alpha)} \\
& +\frac{H_{2}\left(r_{0}, \alpha\right)}{\sigma^{2}(\alpha)} .
\end{aligned}
$$

Therefore, by (4.35),

$$
\begin{aligned}
I_{22} & =\frac{c(1-P)}{2 \pi} \int_{0}^{2 \pi}\left(-\frac{z_{2}}{z_{0} \sigma^{2}(\alpha)}+\frac{H_{1}^{\prime}\left(r_{0}, \alpha\right) H_{1}\left(r_{0}, \alpha\right)}{\sigma^{4}(\alpha) r_{0}(\alpha)}+\frac{H_{2}\left(r_{0}, \alpha\right)}{\sigma^{2}(\alpha)}\right) d \alpha \\
& =\frac{c(1-P)}{2 \pi}\left[-\frac{2 \pi z_{2}}{c z_{0}}+B+C\right] .
\end{aligned}
$$

Thus

$$
\begin{aligned}
I_{2} & =\frac{c(1-P)}{2 \pi} D-(1-P) \frac{z_{2}}{z_{0}}+\frac{c(1-P)}{2 \pi}(B+C) \\
& =-(1-P) \frac{z_{2}}{z_{0}}+\frac{c(1-P)}{2 \pi}(D+B+C),
\end{aligned}
$$

and since $I_{2}=0$, we obtain

$$
z_{2}=\frac{c z_{0}}{2 \pi}(D+B+C)
$$

By (4.40) and (4.41),

$$
\begin{aligned}
r_{2}(\alpha)= & -\frac{z_{2}}{z_{0} \sigma^{2}(\alpha) r_{0}(\alpha)}-\frac{H_{1}^{2}\left(r_{0}, \alpha\right)}{2 \sigma^{2}(\alpha) r_{0}(\alpha)}\left(1+\frac{1}{l}\right) \\
& +\frac{H_{1}^{\prime}\left(r_{0}, \alpha\right) H_{1}\left(r_{0}, \alpha\right)}{\sigma^{4}(\alpha) r_{0}^{2}(\alpha)}+\frac{H_{2}\left(r_{0}, \alpha\right)}{\sigma^{2}(\alpha) r_{0}(\alpha)} \\
= & r_{0}(\alpha) R_{2}(\alpha) .
\end{aligned}
$$

The theorem is proved.

4.3. Proof of Theorem 3.2. It is similar to that of Theorem 3.1. The only difference is that in (4.22) in place of the set $A$ we apply the simpler set $\left\{t \in \mathbb{R}^{2}: \varphi_{\mathbf{0}, W}(t) \geq \bar{t}_{P}\right\}$ (see (3.16)).

4.4. Proof of Theorem 3.3. Observe that

$$
\frac{R\left(\theta, B_{n}\right)}{R\left(\theta, B_{n, 0}\right)}=\frac{\lambda\left(A_{n}\right)}{\lambda\left(A_{n, 0}\right)}=\frac{\lambda\left(A_{n, 0}\right)+\lambda\left(A_{n} \backslash A_{n, 0}\right)-\lambda\left(A_{n, 0} \backslash A_{n}\right)}{\lambda\left(A_{n, 0}\right)}
$$

Define

$$
\begin{aligned}
& \bar{r}^{(n)}(\alpha)=\frac{r_{0}(\alpha)}{\sqrt{n}}\left(1+\frac{\bar{R}_{2}}{n}\right)+o\left(n^{-3 / 2}\right), \\
& r^{(n)}(\alpha)=\frac{r_{0}(\alpha)}{\sqrt{n}}\left(1+\frac{R_{1}(\alpha)}{\sqrt{n}}+\frac{R_{2}(\alpha)}{n}\right)+o\left(n^{-3 / 2}\right) .
\end{aligned}
$$


For all sufficiently large $n$ we have

$$
\text { (4.43) } \begin{aligned}
\lambda\left(A_{n, 0}\right) & =\int_{0}^{2 \pi \bar{r}^{(n)}(\alpha)} \int_{0}^{2 \pi} r d r d \alpha=\frac{1}{2 n} \int_{0}^{2 \pi} r_{0}^{2}(\alpha)\left(1+\frac{2 \bar{R}_{2}}{n}\right) d \alpha+o\left(n^{-2}\right) \\
& =\frac{\pi l \sqrt{\operatorname{det} W}}{n}\left(1+\frac{2 \bar{R}_{2}}{n}\right)+o\left(n^{-2}\right) .
\end{aligned}
$$

Set

$$
E=\left\{\alpha \in[0,2 \pi): \bar{r}^{(n)}(\alpha) \leq r^{(n)}(\alpha)\right\}, \quad E^{\mathrm{c}}=[0,2 \pi) \backslash E .
$$

Notice that

$$
\lambda\left(A_{n} \backslash A_{n, 0}\right)=\int_{E} \int_{\bar{r}^{(n)}(\alpha)}^{r^{(n)}(\alpha)} r d r d \alpha=\frac{1}{2} \int_{E}\left[\left(r^{(n)}(\alpha)\right)^{2}-\left(\bar{r}^{(n)}(\alpha)\right)^{2}\right] d \alpha .
$$

In the same way, one can find $\lambda\left(A_{n, 0} \backslash A_{n}\right)$. Moreover,

$$
\begin{aligned}
& \lambda\left(A_{n} \backslash A_{n, 0}\right)-\lambda\left(A_{n, 0} \backslash A_{n}\right) \\
= & \frac{1}{2} \int_{E}\left[\left(r^{(n)}(\alpha)\right)^{2}-\left(\bar{r}^{(n)}(\alpha)\right)^{2}\right] d \alpha-\frac{1}{2} \int_{E^{\mathrm{c}}}\left[\left(\bar{r}^{(n)}(\alpha)\right)^{2}-\left(r^{(n)}(\alpha)\right)^{2}\right] d \alpha \\
= & \frac{1}{2} \int_{0}^{2 \pi}\left[\left(r^{(n)}(\alpha)\right)^{2}-\left(\bar{r}^{(n)}(\alpha)\right)^{2}\right] d \alpha .
\end{aligned}
$$

Observe that

$$
\begin{aligned}
\left(r^{(n)}(\alpha)\right)^{2}-\left(\bar{r}^{(n)}(\alpha)\right)^{2}=\left(r^{(n)}(\alpha)-\bar{r}^{(n)}(\alpha)\right)\left(r^{(n)}(\alpha)+\bar{r}^{(n)}(\alpha)\right) \\
=\frac{r_{0}^{2}(\alpha)}{n}\left(\frac{R_{1}(\alpha)}{\sqrt{n}}+\frac{R_{2}(\alpha)-\bar{R}_{2}}{n}+o\left(n^{-1}\right)\right) \\
\quad \times\left(2+\frac{R_{1}(\alpha)}{\sqrt{n}}+\frac{R_{2}(\alpha)+\bar{R}_{2}}{n}+o\left(n^{-1}\right)\right) \\
=\frac{2 r_{0}^{2}(\alpha) R_{1}(\alpha)}{n^{3 / 2}}+\frac{r_{0}^{2}(\alpha) R_{1}^{2}(\alpha)+2 r_{0}^{2}(\alpha)\left(R_{2}(\alpha)-\bar{R}_{2}\right)}{n^{2}}+o\left(n^{-2}\right) .
\end{aligned}
$$

Then (4.44) can be rewritten as

$$
\begin{aligned}
& \lambda\left(A_{n} \backslash A_{n, 0}\right)-\lambda\left(A_{n, 0} \backslash A_{n}\right)=\frac{1}{n^{3 / 2}} \int_{0}^{2 \pi} r_{0}^{2}(\alpha) R_{1}(\alpha) d \alpha \\
&+\frac{1}{2 n^{2}} \int_{0}^{2 \pi}\left[r_{0}^{2}(\alpha) R_{1}^{2}(\alpha)+2 r_{0}^{2}(\alpha)\left(R_{2}(\alpha)-\bar{R}_{2}\right)\right] d \alpha+o\left(n^{-2}\right) \\
&=\frac{1}{n^{3 / 2}} J_{1}+\frac{1}{2 n^{2}} J_{2}+o\left(n^{-2}\right) .
\end{aligned}
$$


By Theorem 3.1, we have

$$
J_{1}=\int_{0}^{2 \pi} r_{0}^{2}(\alpha) R_{1}(\alpha) d \alpha=\int_{0}^{2 \pi} \frac{H_{1}\left(r_{0}, \alpha\right)}{\sigma^{2}(\alpha)} d \alpha=0 .
$$

Using Theorems 3.1 and 3.2, we obtain

$$
\begin{aligned}
J_{2} & =\int_{0}^{2 \pi}\left[\frac{2 H_{1}^{\prime}\left(r_{0}, \alpha\right) H_{1}\left(r_{0}, \alpha\right)}{\sqrt{l} \sigma^{3}(\alpha)}+\frac{2 H_{2}\left(r_{0}, \alpha\right)}{\sigma^{2}(\alpha)}-\frac{H_{1}^{2}\left(r_{0}, \alpha\right)}{\sigma^{2}(\alpha)}-\frac{c(B+C)}{\pi \sigma^{2}(\alpha)}\right] d \alpha \\
& =2 B+2 C-\int_{0}^{2 \pi} \frac{H_{1}^{2}\left(r_{0}, \alpha\right)}{\sigma^{2}(\alpha)} d \alpha-2 B-2 C=-\int_{0}^{2 \pi} \frac{H_{1}^{2}\left(r_{0}, \alpha\right)}{\sigma^{2}(\alpha)} d \alpha .
\end{aligned}
$$

Therefore,

$$
\lambda\left(A_{n} \backslash A_{n, 0}\right)-\lambda\left(A_{n, 0} \backslash A_{n}\right)=\frac{1}{2 n^{2}} J_{2}+o\left(n^{-2}\right)
$$

and (4.42) can be rewritten as

$$
\frac{R\left(\theta, B_{n}\right)}{R\left(\theta, B_{n, 0}\right)}=1+\frac{1}{2 n^{2} \lambda\left(A_{n, 0}\right)} J_{2}+o\left(n^{-2}\right) .
$$

By (4.43), we have

$$
n \lambda\left(A_{n, 0}\right)=\pi l \sqrt{\operatorname{det} W}\left(1+\frac{2 \bar{R}_{2}}{n}\right)+o\left(n^{-1}\right),
$$

which implies

$$
\frac{1}{n \lambda\left(A_{n, 0}\right)}=\frac{1}{\pi l \sqrt{\operatorname{det} W}}\left(1-\frac{2 \bar{R}_{2}}{n}\right)+o\left(n^{-1}\right) .
$$

Since

$$
\frac{R\left(\theta, B_{n}\right)}{R\left(\theta, B_{n, 0}\right)}-1=\frac{J_{2}}{2 n \pi l \sqrt{\operatorname{det} W}}\left(1-\frac{2 \bar{R}_{2}}{n}\right)+o\left(n^{-2}\right),
$$

it follows that

$$
\begin{aligned}
\frac{R\left(\theta, B_{n, 0}\right)}{R\left(\theta, B_{n}\right)}-1 & =-\frac{J_{2}}{2 \pi n l \sqrt{\operatorname{det} W}}+o\left(n^{-1}\right) \\
& =\frac{1}{2 \pi n l \sqrt{\operatorname{det} W}} \int_{0}^{2 \pi} \frac{H_{1}^{2}\left(r_{0}, \alpha\right)}{\sigma^{2}(\alpha)} d \alpha+o\left(n^{-1}\right) .
\end{aligned}
$$

The theorem is proved.

Acknowledgements. We wish to thank the referee for his careful reading of the manuscript and for valuable comments. 


\section{References}

R. Bartoszyński and W. Chan (1990), A remark on the shortest confidence interval of a normal mean, Amer. Math. Monthly 97, 415-417.

R. N. Bhattacharya and R. Ranga Rao (1976), Normal Approximation and Asymptotic Expansions, Wiley, New York.

A. Czarnowska and A. V. Nagaev (2001), Confidence regions of minimal area for the scale-location parameter and their applications, Appl. Math. (Warsaw) 28, 125-142.

J. H. J. Einmahl and D. M. Mason (1992), Generalized quantile processes, Ann. Statist. 20, 1062-1078.

J. N. Garcia, Z. Kutalik, K.-H. Cho and O. Wolkenhauer (2003), Level sets and minimum volume sets of probability density functions, Internat. J. Approx. Reason. 34, 25-47.

W. C. Guenther (1969), Shortest confidence intervals, Amer. Statist. 23, 22-25.

S. Jeyaratnam (1985), Minimum volume confidence regions, Statist. Probab. Lett. 3, 307308.

W. Polonik (1997), Minimum volume sets and generalized quantile processes, Stochastic Process. Appl. 69, 1-24.

J. W. Pratt (1961), Length of confidence intervals, J. Amer. Statist. Assoc. 56, 549-567.

S. M. Sadikova (1966), Some inequalities for characteristic functions, Theory Probab. Appl. 11, 500-506.

T. Tonda and H. Wakaki (2003), Asymptotic expansion of the null distribution of the likehood ratio statistic for testing the equality of variances in a nonnormal one-way ANOVA model, Hiroshima Math. J. 33, 113-126.

Faculty of Mathematics and Computer Science

Nicolaus Copernicus University

Chopina 12/18

87-100 Toruń, Poland

E-mail: plamka@mat.uni.torun.pl

alzaig@mat.uni.torun.pl

Received on 21.6.2005;

revised version on 19.1.2006 